\title{
Uma alternativa para avaliar a eficiência de meios de cultivo in vitro via modelo de regressão logística
}

\section{An alternative to evaluate the efficiency of in vitro culture medium using a logistic regression model}

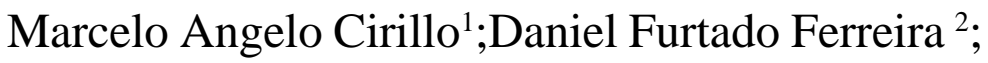 \\ Patrícia Schober Gonçalves Lima ${ }^{3}$
}

\begin{abstract}
Resumo
A avaliação do meio de cultura para o cultivo in vitro de uma determinada espécie é feita através de propriedades físicas e/ou químicas inerentes ao meio de cultivo. No entanto, a análise de resultados experimentais permitem avaliar sobre a qualidade deste meio. Nesse sentido, este trabalho apresenta uma alternativa, por meio da utilização de um modelo logístico para avaliar o meio de cultura a ser utilizado no cultivo in vitro. As probabilidades fornecidas por este modelo servirão como um "índice avaliador do meio". A importância desse índice é dada em função da formalização de um critério estatístico para a seleção do meio de cultura adequado a ser utilizado no cultivo in vitro, não excluindo as propriedades físicas e/ou químicas do mesmo. Para a ilustração desse procedimento, foi avaliado um experimento no qual se determinou o meio de cultura ideal no cultivo in vitro dos explantes primários retirados da Ipeca (Psychotria ipecacuanha (Brot.) Stokes). A diferenciação destes meios foi dada pela ausência ou presença do hormônio de crescimento BAP (6-benzilaminopurina). Ajustou-se um modelo logístico em função das covariáveis caracterizadas pelos pesos de matéria fresca e seca. As probabilidades mínima, média e máxima obtidas deste modelo mostraram que o meio de cultivo no qual o BAP (6benzilaminopurina) estava presente foi o mais propício para a reprodução dos explantes. Devido ao alto poder discriminativo destes meios, detectado pelo modelo, recomenda-se a sua utilização como alternativa para seleção de meio de cultura para experimentos em situações semelhantes no cultivo in vitro.

Palavras-chave: in vitro, modelo logístico, probabilidades.
\end{abstract}

\begin{abstract}
The evaluation of a culture medium for the in vitro culture of a species is performed using its physical and/or chemical properties. However, the analysis of the experimental results makes it possible to evaluate its quality. In this sense, this work presents an alternative using a logistic model to evaluate the culture medium to be used in vitro. The probabilities provided by this model will be used as a medium evaluator index. The importance of this index is based on the formalization of a statistical criterion for the selection of the adequate culture medium to be used on in vitro culture without excluding its physical
\end{abstract}

\footnotetext{
Doutorando em Estatística Experimentação Agropecuária, Departamento de Ciências Exatas - UFLA, Bolsista: FAPEMIG. Prof. Adjunto do Departamento de Ciências Exatas - UFLA, Bolsista: CNPq na modalidade de produtividade em pesquisa. 3 Mestre em Genética/Melhoramento Vegetal - Departamento de Biologia - UFLA
} 
and/or chemical properties. To demonstrate this procedure, an experiment determining the ideal medium for the in vitro culture of primary explants of Ipeca [Psychotria ipecacuanha (Brot.) Stokes] was evaluated. The differentiation of the culture medium was based on the presence and absence of the growth regulator BAP (6-benzilaminopurine). A logistic model was adjusted as a function of the weight of fresh and dry matter. Minimum, medium and maximum probabilities obtained with this model showed that the culture medium containing BAP was the most adequate for the explant growth. Due to the high discriminative power of these mediums, detected by the model, their use is recommended as an alternative to select culture medium for similar experiments.

Key words: in vitro, logistic model, probabilities.

\section{Introdução}

Uma das vertentes da pesquisa na área de melhoramento vegetal refere-se à propagação $\mathrm{e}$ conservação de uma determinada espécie. Entretanto, em muitas situações de estudo, o trabalho científico se depara com dificuldades, tais como, o fato de algumas plantas só conseguirem desenvolver-se em condições ecológicas específicas. Para contornar esse problema, em geral são utilizadas técnicas de cultura de tecido in vitro, a qual consiste em isolar qualquer parte da planta, definida como material vegetal para cultivo em condições de laboratório sobre um meio nutritivo artificial (meio de cultura).

O material vegetal é representativo de qualquer parte da planta, seja uma célula, um órgão ou um tecido. A propagação deste material é feita em tubos de ensaio ou material similar, em adequadas condições de assepsia, nutrição e fatores ambientais. $\mathrm{O}$ uso desses objetos (p.ex. tubos de ensaio) sugere a expressão in vitro.

O uso da técnica do cultivo in vitro pode propiciar inúmeras vantagens: entre as principais, pode-se citar a rápida propagação da espécie. No caso de plantas medicinais, que só prosperam sob determinadas condições ecológicas, o tempo para obtenção de mudas pode ser reduzido, diminuindo assim o custo de produção.

Segundo Grattapaglia e Machado (1998), a cultura in vitro, também denominada de micropropagação, é a aplicação de maior impacto da cultura de tecidos dentro da agricultura. $\mathrm{O}$ sucesso de um sistema de micropropagação é altamente dependente do meio de cultura, cuja composição física e química é previamente definida por um balanço nutricional e hormonal. Neste contexto, Nannetti (1994) salientou o nitrogênio como um importante mineral, cuja combinação das formas amônia e nitrato estimulam o crescimento das plantas in vitro. Em relação ao aspecto hormonal, os reguladores de crescimento presentes no meio de cultura direcionam não só o desenvolvimento da planta, mas também o sucesso do cultivo. Entre os reguladores de crescimento utilizado no cultivo in vitro, Gonçalves (2002) afirma que o BAP (6benzilaminopurina) é a que, em geral, apresenta melhores resultados

Uma variedade de experimentos in vitro é realizada na cultura de tecidos vegetais; em todos eles faz-se necessário o uso de técnicas estatísticas para a validação dos resultados experimentais. Entre as técnicas utilizadas, os modelos de regressão são amplamente empregados. Andrade et al. (2001) utilizaram um modelo de regressão em uma superfície de reposta, com o objetivo de avaliar o número total de brotos em embriões de cafeeiro, submetidos a diferentes concentrações dos reguladores de crescimento NAA (ácido naftalenacético) e BAP(6-benzinamilapurina). Costa et al. (2002) utilizaram modelos de regressão linear simples para avaliar a influência da concentração de soluções nutritivas no acúmulo de biomassa na parte aérea das plantas da ipeca.

Para as situações mencionadas, as respostas dos experimentos apresentaram uma característica comum, ou seja, os valores eram dados em escala contínua, o que favorece o uso de modelos de regressão com os erros independentes e normalmente 
distribuídos. No entanto, dependendo do interesse do pesquisador, a resposta do experimento pode ser dada em uma escala discreta, como, por exemplo, uma resposta binária caracterizando sucesso ou fracasso.

No contexto da cultura de tecido, pode-se exemplificar essa situação por meio da resposta da formação ou não de muda de um determinado explante, em que o sucesso é dado pela formação de muda. Decorrente dessa característica na resposta, o uso de um modelo de regressão linear simples, representado por $\mathrm{Y}_{\mathrm{i}}=\mathrm{b}_{0}+\mathrm{b}_{1} \mathrm{X}_{\mathrm{i}}+\mathrm{e}_{\mathrm{i}}$, não é aplicável, pois supõe-se que os resíduos definidos por $e_{i}=Y_{i}-b_{0}-b_{1} X_{i}$ são independentes e normalmente distribuídos com média zero e variância $\mathrm{s}^{2}$. No caso da regressão logística com dados binários, a variável resposta segue a distribuição de Bernoulli. Os resíduos serão dados apenas por dois possíveis resultados:

$$
\mathrm{e}_{\mathrm{i}}=1-\left(\mathrm{b}_{0}-\mathrm{b}_{1} \mathrm{X}_{\mathrm{i}}\right) \text { ou } \mathrm{e}_{\mathrm{i}}=-\mathrm{b}_{0}-\mathrm{b}_{1} \mathrm{X}_{\mathrm{i}} \text {. }
$$

Dessa forma, os resíduos não serão normalmente distribuídos conforme mencionado por Costa (1997).

Para análise de dados dessa natureza, são necessárias técnicas estatísticas condizentes com este tipo de resposta. Entre as técnicas utilizadas aplicamse modelos generalizados ou regressão logística (BALAKRISHNAN, 1991).

Como foi mencionado anteriormente, a eficiência da cultura in vitro é essencialmente determinada pelo meio de cultura. A avaliação deste meio é feita basicamente em suas propriedades físicas e/ou químicas, além de inúmeros fatores e peculiaridades inerentes às diversas espécies. Em virtude do que foi mencionado, este trabalho objetivou apresentar uma alternativa para a avaliação de meios de cultivo in vitro. Para ilustração deste procedimento, foram utilizados os pesos de matéria fresca e seca provenientes de um experimento, o qual consistiu em determinar qual o meio de cultura ideal no cultivo in vitro dos explantes primários retirados da planta arbustiva [Psychotria ipecacuanha (Brot.) Stokes] conhecida como ipeca. Os meios de cultura foram diferenciados pela ausência e presença de BAP(6benzinamilapurina).

\section{Material e Métodos}

Este trabalho foi realizado no Laboratório de Cultura de Tecidos Vegetais e Plantas Medicinais da Universidade Federal de Lavras (UFLA). O material vegetal utilizado consistiu de plântulas provenientes de segmentos internodais retirados da ipeca. As plântulas foram cultivadas em dois meios de cultura, sendo estes diferenciados pela ausência ou presença de BAP (6-benzilaminopurina). Os tubos de ensaio (25 $\mathrm{X} 150 \mathrm{~mm})$ em que as plântulas foram inoculadas continham $30 \mathrm{ml}$ do meio de cultura. Nessas condições, tomou-se uma amostra de 173 exemplares.

Devido a vários fatores que influenciam o desenvolvimento da plântula na formação de muda, tais como luminosidade, meio de cultivo e contaminação por fungos, foi analisada a viabilidade da plântula produzir a muda em função do peso da matéria fresca e seca obtidos com auxílio de uma balança de precisão.

O peso da matéria fresca é acrescido de água e a medição é feita em gramas no momento em que a muda formada saiu do tubo. O peso da matéria seca foi obtido por secagem em uma estufa a $70^{\circ} \mathrm{C}$ durante 48 horas.

Para avaliar estatisticamente a eficiência do meio de cultura, considerou-se como resposta de interesse o fato da plântula ser viável ou não, isto é, ter gerado ou não mudas. Essas características foram codificadas em uma variável aleatória $\mathrm{Y}$ com distribuição de Bernouli, de tal forma que $Y=1$ corresponde a plântula ter gerado muda e $\mathrm{Y}=0$, a plântula não ter gerado a muda. Sendo assim, ajustouse um modelo logístico (2) para a característica de interesse, ou seja, a plântula ter gerado muda $(Y=1)$ em função das covariáveis PF e PS representando os pesos de matéria fresca e seca.

Representando as observações das covariáveis PF e PS, respectivamente por $\mathrm{X}_{1 \mathrm{i}}$ e $\mathrm{X}_{2 \mathrm{i}}$, inicialmente foi realizada uma análise preliminar entre $\mathrm{o}$ comportamento destas variáveis. Para realização desse procedimento, inicialmente foi obtida a 
correlação entre $X_{1 i}$ e $X_{2 i}$, uma vez que o peso da matéria seca (PS) é condicionado ao peso da matéria fresca (PF). Para isso utilizou-se a correlação de Pearson (BUSSAB; MORETTIN, 1987) dada pela expressão (1)

$$
\hat{\rho}_{\mathrm{X}_{\mathrm{li}} \mathrm{X}_{2 \mathrm{i}}}=\frac{\sum_{\mathrm{i}=1}^{\mathrm{n}}\left(\mathrm{X}_{1 \mathrm{i}}-\overline{\mathrm{X}}_{1}\right)\left(\mathrm{X}_{2 \mathrm{i}}-\overline{\mathrm{X}}_{2}\right)}{\sqrt{\sum_{i=1}^{n}\left(\mathrm{X}_{1 \mathrm{i}}-\bar{X}_{1}\right)^{2} \sum_{i=1}^{n}\left(\mathrm{X}_{2 \mathrm{i}}-\bar{X}_{2}\right)^{2}}}
$$

Após esta análise preliminar, procedeu-se ao ajuste do modelo logístico (2), de acordo com as características de cada variável, mencionadas anteriormente. Sendo assim, o modelo ajustado é dado por:

$$
\mathrm{P}(\mathrm{Y}=1 \mid \mathrm{PF}, \mathrm{PS})=\frac{1}{1+e^{-\left(\hat{\mathrm{a}}_{0}+\hat{a}_{1} X_{1 \mathrm{i}}+\hat{a}_{2} X_{2 i}\right)}},
$$

em que $X_{1 \mathrm{i}}$ e $X_{2 \mathrm{i}}$ representam os pesos da matéria seca e fresca, $\beta_{1}$ e $\beta_{2}$ são os coeficientes estimados para os respectivos pesos, e $\beta_{0}$ corresponde ao intercepto do termo linear presente na expressão (2). Dessa forma, considerando-se os pesos da matéria fresca e seca conhecidos para cada plântula, estimaram-se os parâmetros $\beta_{0}, \beta_{1}$ e $\beta_{2}$ do termo linear por meio da transformação logito apresentada por Allison (1999), conforme a expressão (3):

$$
\log \left[\frac{p_{i}}{1-p_{i}}\right]=\hat{a}_{0}+\hat{a}_{1} X_{1 i}+\hat{a}_{2} X_{2 i}
$$

em que $\mathrm{p}_{\mathrm{i}}$ representa a probabilidade de cada plântula ter gerado a muda. Com base no modelo logístico ajustado, foram realizadas inferências relacionadas à qualidade das estimativas dos parâmetros. Para isso, utilizou-se o teste Qui-Quadrado de Wald, conforme a estatística (4).
$Z=\frac{\hat{\hat{a}}_{i}}{S_{E_{i}}}$,

em que $\mathrm{S}_{\mathrm{Ei}}$ corresponde ao erro padrão do i-ésimo coeficiente estimado.

Para verificar a qualidade do ajuste do modelo, utilizou-se a deviance definida por

$$
\mathrm{D}=-2 \sum_{\mathrm{i}=1}^{\mathrm{n}}\left[\mathrm{y}_{\mathrm{i}} \ln \left(\hat{\mathrm{p}}_{\mathrm{i}}\right)+\left(1-\mathrm{y}_{\mathrm{i}}\right) \ln \left(1-\hat{\mathrm{p}}_{\mathrm{i}}\right)\right]
$$

em que $\hat{\mathrm{p}}_{\mathrm{i}}$ representa o i-ésimo valor ajustado para a i-ésima observação $\left(\mathrm{y}_{\mathrm{i}}\right)$.

A importância da análise da deviance é primordial para detectar o efeito da superdispersão. A ocorrência desse efeito implica em uma variação observada, superior à variação assumida pelo modelo. A ocorrência deste efeito é comum na modelagem de dados binários, devido a diferentes circunstâncias (MCCULLAGH; NELDER, 1992).

A avaliação da predição do modelo ajustado foi feita por meio da correlação entre as probabilidades preditas e a resposta observada $(\mathrm{Y}=1$ ou $\mathrm{Y}=0)$; conforme mencionado anteriormente, esses valores referem-se respectivamente a plântula ter gerado ou não muda da ipeca. De acordo com estas duas respostas, o número total de pares que poderão ser formados, combinando-se as probabilidades preditas e a resposta observada, é $\mathrm{C}_{2}^{\mathrm{n}}$. A determinação se o par de observação é concordante ou discordante baseia-se na comparação de todos os pares que apresentam $(\mathrm{Y}=0)$ com o evento de interesse $(\mathrm{Y}=1)$. Considerando-se o modelo ajustado, a resposta observada $(\mathrm{Y}=0)$ que apresentar menor probabilidade em relação a resposta observada $(Y=1)$, classificase esse par $(\mathrm{Y}=1, \mathrm{Y}=0)$ como concordante. $\mathrm{O}$ inverso dessa situação caracteriza o par como discordante. Se as probabilidades preditas forem iguais, os pares são classificados como empate. Seguindo esta classificação os coeficientes de correlação 
apresentados por Allison (1999) para medir esta correlação são descritos pelas expressões (6), (7) e (8)

Kendal Tau-a $=\frac{\mathrm{C}-\mathrm{D}}{\mathrm{N}}$;

Goodman Kruskal - Gamma $=\frac{C-D}{C+D}$;

Somer's $\mathrm{D}=\frac{\mathrm{C}-\mathrm{D}}{\mathrm{C}+\mathrm{D}+\mathrm{T}}$

em que $\mathrm{C}=$ número total de pares concordantes, $\mathrm{D}=$ número total de pares discordantes, $\mathrm{T}=$ número total de pares que ocasionaram empates e $\mathrm{N}$ representa o número total de pares. Caso os valores das correlações, provenientes de pelo menos dois métodos, sejam próximos a 1 , pode-se concluir que o modelo ajustado apresenta alto poder preditivo.

Um outro fator que deve ser considerado para a validação do modelo logístico é dado em função da sensibilidade do modelo ajustado às observações influentes. Em virtude dessa sensibilidade, procurouse identificar a influência das observações na estimação dos parâmetros. Para avaliação desta influência, foram utilizados como medida os valores

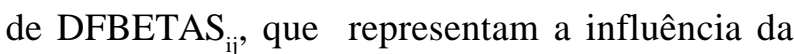
retirada da i-ésima observação para o j-ésimo coeficiente do modelo, conforme a expressão (9).

$\operatorname{Dfbetas}_{\mathrm{ij}}=\frac{\hat{\hat{a}}_{\mathrm{j}} \hat{\hat{\mathrm{a}}}_{\mathrm{j}(\mathrm{i})}}{\mathrm{s}_{(\mathrm{i})} \sqrt{\mathrm{a}_{\mathrm{j} j}}}$,

em que $\mathrm{a}_{\mathrm{jij}}$ refere-se à diagonal da matriz $\left(\mathrm{X}^{\prime} \mathrm{X}\right)^{-1}$ e $\mathrm{s}_{(\mathrm{i})}$ corresponde ao i-ésimo erro padrão do j-ésimo coeficiente. De acordo com Souza (1998), os pontos são considerados influentes, caso o valor absoluto obtido por esta medida obedeça à relação (10)

$\mid$ Dfbetas $_{\mathrm{ij}} \mid>\frac{2}{\sqrt{\mathrm{n}}}$,

em que $\mathrm{n}$ representa o tamanho amostral.
Após a estimação do modelo logístico e avaliação do mesmo em função da significância de seus parâmetros, análise dos valores preditos e análise das observações influentes, este foi validado em função da aplicação em outro meio de cultivo, sendo os resultados comparados.

\section{Resultados e Discussão}

É sabido que na situação de uma forte correlação há evidências de que a validação do modelo poderia ser influenciada e, desejando-se verificar o grau de correlação entre as variáveis (PF) e (PS), fez-se necessário estimar a correlação de Pearson, cujo valor foi de 0,5565 . O fato desse valor ter caracterizado uma média correlação leva a crer que os resultados experimentais relativos a essas variáveis são independentes. É importante ressaltar que esta correlação apresenta apenas um resultado exploratório, pois não incorpora informações a respeito da variável de interesse (formação ou não de mudas). Sendo assim, faz-se necessário enriquecer a análise estatística destas variáveis com métodos inferênciais, cujos resultados são apresentados a seguir.

Inicialmente, ajustou-se o modelo logístico em função dos pesos da matéria seca (PS) e da matéria fresca $(\mathrm{PF})$ por meio da transformação logito. A este modelo ajustado denominou-se modelo completo, em virtude de todos os parâmetros estarem contidos nele. Conforme os resultados encontrados na Tabela 1 , verificou-se, por meio do teste Qui-Quadrado de Wald, que o efeito do peso da matéria fresca (PF) e o intercepto contribuíram significativamente no ajuste deste modelo $(\mathrm{p}<0,05)$. Porém, o peso da matéria seca (PS) foi não significativo ( $p>0,05$ ), ou seja, estatísticamente o coeficiente estimado para este parâmetro é igual a zero, portanto, caracteriza uma elevada redundância dessa variável no ajuste do modelo.

Devido a esse problema, foi ajustado um novo modelo, excluindo a variável (PS). A este novo modelo ajustado denominou-se de modelo reduzido. As estimativas dos modelos completo e reduzido são encontradas na Tabela 1. 
Tabela 1 - Estimativas dos parâmetros e probabilidades do teste Wald para os modelos completos e reduzidos.

\begin{tabular}{cccccc}
\hline \multicolumn{3}{c}{ Modelo completo } & \multicolumn{3}{c}{ Modelo reduzido } \\
\hline Parâmetro & Coeficiente & p-valor & Parâmetro & Coeficiente & p-valor \\
& & & & & \\
\hline Intercepto & $-2,720$ & 0,0001 & Intercepto & $-2,684$ & 0,0001 \\
PF & 50,736 & 0,0001 & PF & 47,925 & 0,0001 \\
PS & $-8,0109$ & 0,3187 & & & \\
\hline
\end{tabular}

Os resultados apresentados anteriormente por meio da Tabela 1 evidenciam que a variável PS pode ser excluída da análise, e indicam preliminarmente que o uso do modelo reduzido fosse adequado. Apesar disso, deve ser ressaltada a importância dessa variável no contexto experimental, ou seja, no decorrer do experimento, foi relevante comportamento da variável peso da matéria seca para o desenvolvimento das plântulas que ocasionaram a formação de mudas . Isso pode ser constatado por meio da Figura 1, na qual se verifica que o peso da matéria seca foi importante para a formação de mudas, principalmente para os pesos inferiores a 0,05g. Em virtude desse resultado, justifica-se a comparação entre os modelos completo e reduzido, não apenas por satisfazer às pré-suposições estatísticas, tais como estabilização dos resíduos e análise de observações discrepantes, mas também considerando-se os resultados experimentais.

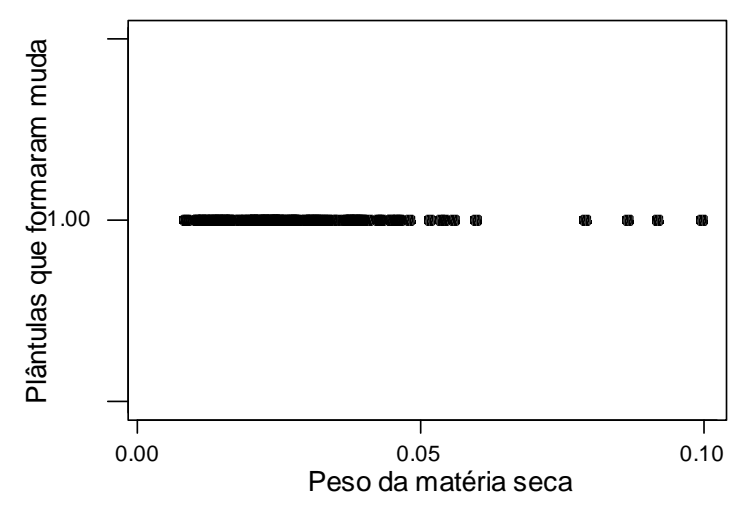

Figura 1 - Performance do peso da matéria seca nas plântulas que ocasionaram a formação de mudas.
Justificada a comparação dos modelos completo e reduzido, procedeu-se à análise estatística, verificando-se o efeito da superdispersão utilizandose a análise da Deviance. De acordo, com os resultados mostrados na Tabela 2, com base nas probabilidades obtidas da distribuição de Quiquadrado, concluiu-se que, em ambos modelos, não existe o efeito da superdispersão ( $p$-valor $>0,05$ ). A ausência deste efeito condiz com a validação do modelo ajustado mediante os dados observacionais, mostrando que os modelos são adequados e estão bem ajustados.

É importante ressaltar que o efeito da superdispersão pode ser ocasionado por vários fatores, entre os quais pode-se salientar a presença de observações discrepantes ou a ausência de algum parâmetro no modelo. Em decorrência desse último fator, justificou-se a análise da deviance para o modelo reduzido, sendo assim, pode-se verificar que a redução do parâmetro PS não ocasionou este efeito. Aparentemente, essa característica favorece a escolha do modelo reduzido por se tratar de um modelo com um número menor de parâmetros e também pela adequação aos dados.

Tabela 2 - Análise da Deviance para validade do ajuste dos modelo logístico completo e reduzido.

\begin{tabular}{lccc}
\hline & $\begin{array}{c}\text { Valor Qui- } \\
\text { Quadrado }\end{array}$ & $\begin{array}{c}\text { Grau de } \\
\text { liberdade }\end{array}$ & p-valor \\
\hline Modelo Completo & 131,52 & 170 & 0,987 \\
Modelo Reduzido & 121,26 & 160 & 0,990 \\
\hline
\end{tabular}

A qualidade do ajuste do modelo foi verificada por meio da correlação entre as probabilidades estimadas e a resposta observada. Dessa forma, verificou-se, por meio dos resultados encontrados na Tabela 3, que as medidas de correlação Somers'D e Goodmam Kruskal-Gamma apresentaram valores aproximados entre si e próximo a 1. Portanto, podese concluir que os valores preditos estão 
correlacionados com os valores observados, ou seja, a predição de ambos os modelos são coerentes com os valores observados. Entretanto, a análise da correlação de Kendal Tau-a permite verificar que o valor da correlação foi baixo. Segundo Zimmermann (1989), a distribuição do coeficiente de correlação de Kendal se aproxima da distribuição normal e, no caso da regressão logística a variável de interesse, $Y$ possui uma distribuição de Bernoulli, isto é, assume apenas dois valores 1 ou 0 , portanto, sua escala é discreta o que justifica a baixa correlação.

Tabela 3 - Medidas descritivas e coeficientes de correlação entre as probabilidades estimadas e resposta observada.

\begin{tabular}{cc}
\hline \multicolumn{2}{c}{ Modelo Completo } \\
\hline Medidas descritivas & Coeficiente de correlação \\
\hline Pares Concordantes $=88,6 \%$ & Somers' D = 0,773 \\
Pares Discordantes $=11,3 \%$ & Goodmam Kruskal - Gamma $=0,773$ \\
Pares Empatados $=0,01 \%$ & Kendal Tau-a = 0,319 \\
\hline \multicolumn{1}{c}{ Modelo Reduzido } \\
\hline Medidas descritivas & Coeficiente de correlação \\
\hline Pares Concordantes $=88,4 \%$ & Somers' D = 0,769 \\
Pares Empatados $=0,01 \%$ & Goodmam Kruskal - Gamma $=0,770$ \\
\hline
\end{tabular}

A análise de observações discrepantes foi realizada com o objetivo de verificar quais observações foram influentes na estimação individual de cada parâmetro. Dessa forma, identificada as observações influentes e confrontando-as com os modelos em estudo, tem-se um critério adicional para a escolha do modelo a ser utilizado, propiciando predições mais precisas.

Outra vantagem que essa análise apresenta é a confirmação da importância do parâmetro relacionado à variável PS na estimativa dos coeficientes. A medida relacionada a este tipo de análise é referenciada por Souza (1998) como DFBETAS, de modo que os pontos que são considerados influentes são aqueles cujos valores estimados para o DFBETA ultrapassam o limite de tolerância dado por 0,1520 . Para melhor visualizar quais observações foram influentes, plotaram-se os DFBETAS versus as observações em conjunto com o limite especificado. Os resultados são apresentados nas Figuras 2-5.

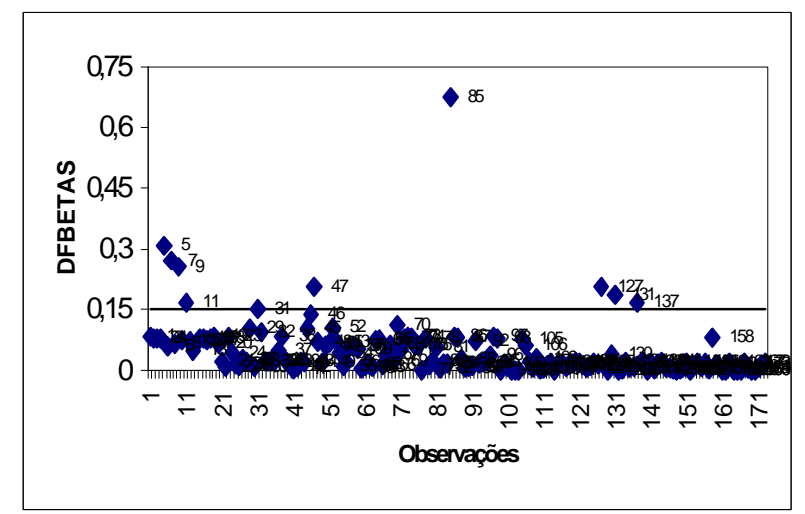

Figura 2- Valores DFBETAS para o intercepto ajustado ao modelo completo.

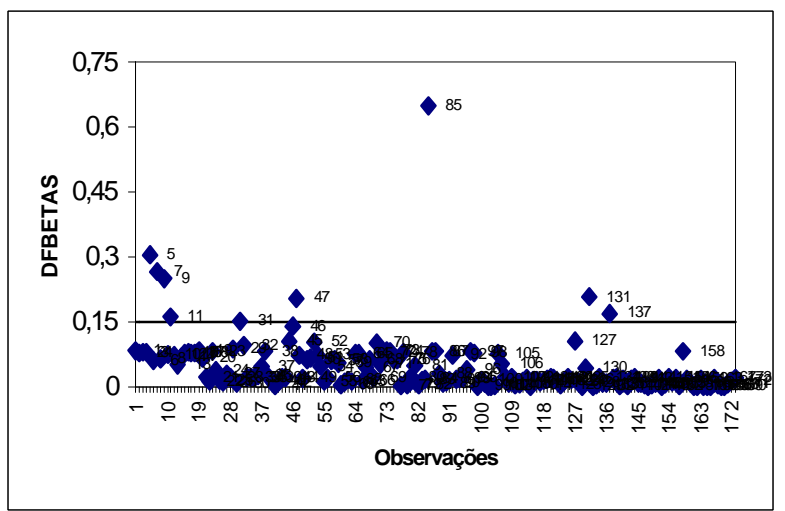

Figura 3 - Valores DFBETAS para o intercepto ajustado ao modelo reduzido.

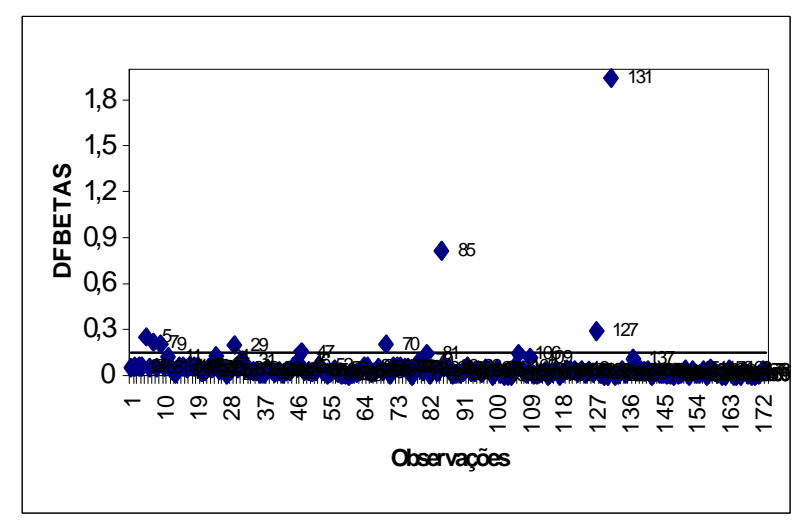

Figura 4 - Valores DFBETAS para a variável PF ajustada ao modelo completo. 


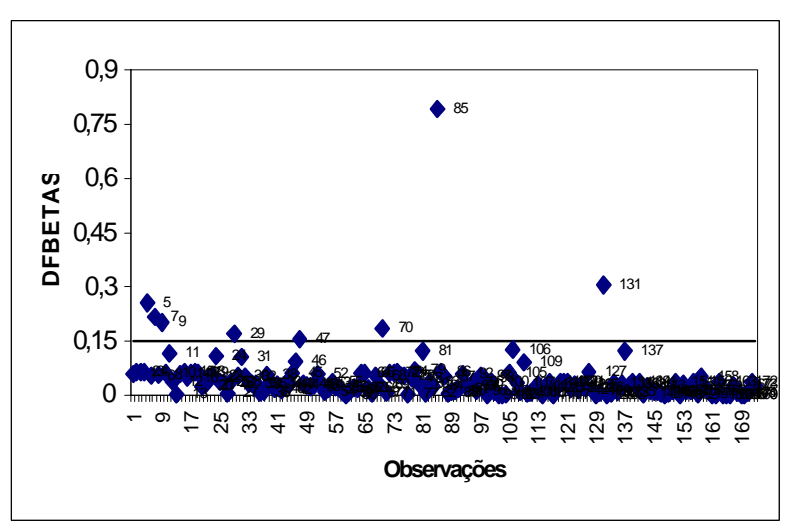

Figura 5 - Valores DFBETAS para a variável PF ajustada ao modelo reduzido

Em relação ao intercepto, os resultados apresentados pelas Figuras 2 e 3 evidenciaram que os modelos estudados apresentaram praticamente os mesmos pontos considerados como influentes na estimação do intercepto, exceto a observação $n^{\circ} 127$, que no modelo reduzido foi considerada não influente. Dessa forma, conclui-se que a adição ou não da variável que representa PS não interferiu na estimação do intercepto.

Em relação à variável $\mathrm{PF}$, os resultados apresentados pelas Figuras 4 e 5 mostraram a mesma evidência em relação à adição ou não da variável PS, pois foi detectado, praticamente o mesmo número de observações influentes na estimação do parâmetro PF.

Após ter realizado todos os procedimentos convencionais para validação de um modelo estatístico, verificou-se que, teoricamente, ambos os modelos poderiam ser usados para explicar a formação de mudas resultante do cultivo de plântulas. Entretanto, não se tem nenhuma informação que relacione a qualidade do meio de cultivo na formação de mudas, resultante do desenvolvimento de plântulas no cultivo in vitro. Em função desse fato, a determinação de qual modelo deve ser utilizado como medidor da eficiência do meio de cultura na formação das mudas foi dada em função das probabilidades fornecidas. Dessa forma, a maior probabilidade média obtida indicou qual modelo deve ser utilizado para a determinação do índice de eficiência do meio de cultura. Para isso, calcularamse as probabilidades mínima, média e máxima para os modelos completo e reduzido sobre dois meios de cultivo, diferenciados pela ausência ou presença do BAP(6-benzilaminopurina). Os resultados obtidos estão apresentados na Tabela 4.

Tabela 4 - Avaliação do meio de cultura para mudas de ipeca provenientes de plântulas cultivadas in vitro

\begin{tabular}{cccc}
\hline Meio de cultivo & $\begin{array}{c}\text { Probabilidade } \\
\text { mínima }\end{array}$ & $\begin{array}{c}\text { Probabilidade } \\
\text { média }\end{array}$ & $\begin{array}{c}\text { Probabilidade } \\
\text { Máxima }\end{array}$ \\
\hline $\begin{array}{c}\text { Com BAP } \\
\text { (Modelo completo) }\end{array}$ & 0,1836 & 0,8265 & 0,9999 \\
$\begin{array}{c}\text { Sem BAP } \\
\text { (Modelo completo) }\end{array}$ & 0,0010 & 0,0682 & 0,3873 \\
\hline $\begin{array}{c}\text { Com BAP } \\
\text { (Modelo reduzido) }\end{array}$ & 0,1888 & 0,8257 & 0,999 \\
$\begin{array}{c}\text { Sem BAP } \\
\text { (Modelo reduzido) }\end{array}$ & 0,1605 & 0,5978 & 1,000 \\
\hline
\end{tabular}

Pela análise das probabilidades médias, mínimas e máximas de ambos os modelos em dois diferentes meios de cultivo (Tabela 4), observou-se que o modelo completo e o modelo reduzido apresentaram resultados praticamente idênticos na discriminação dos meios de cultivo com BAP, situação na qual foram ajustados. Isso significa que ambos os modelos têm o mesmo potencial discriminativo em selecionar o meio de cultivo ideal, ou seja, o meio de cultivo no qual esta adicionado o regulador BAP. Diante dessa situação, poderia ser utilizado o critério de parcimoniosidade e adotar-se o modelo mais simples (modelo reduzido). No entanto, quando são comparados os modelos no meio sem BAP em relação às probabilidades máxima, média e mínima, verificou-se que o modelo reduzido apresentou valores mais elevados. Se forem comparadas as probabilidades mínimas, médias e máximas dentro de cada modelo para os dois meios de cultivo, podese observar que o modelo completo apresentou um poder discriminativo maior do que o do modelo reduzido. Isso se dá pelas maiores amplitudes entre as probabilidades encontradas no meio de cultivo com BAP em relação ao meio de cultivo sem BAP do modelo completo. 
O modelo completo ajustado é apresentado a seguir, para que outros pesquisadores possam utilizálo na discriminação de meios de cultivo. Essa discriminação deve ser feita comparando-se as probabilidades mínimas, médias e máximas de uma amostra submetida a esses meios que se deseja comparar. Dessa forma, pode-se resumir a utilização prática deste modelo em responder à seguinte pergunta:

"Conhecidos os pesos da matéria seca e da matéria fresca, qual a probabilidade de que a plântula seja viável, isto é, apresente condições de gerar uma muda em um determinado meio de cultivo?"

$\mathrm{P}(\mathrm{Plân}$ tula ser viavel $=$ Sim $\mid \mathrm{PF}, \mathrm{PS})=\frac{1}{1+\exp (2,7204-50,736 \mathrm{PF}+8,0109 \mathrm{PS})}$

\section{Conclusões}

Os pesos da matéria seca e fresca são primordiais para avaliação do meio de cultivo via modelo de regressão logística.

O modelo logístico, em função dos pesos de matéria fresca e seca, apresentou alto poder discriminativo entre os meios de cultura. Portanto, considerou-se uma alternativa mais adequada para sua aplicação na seleção do meio de cultura.

O procedimento aplicado neste trabalho pode ser utilizado em situações de modelagem semelhantes, de tal forma que os resultados obtidos poderão agregar informações na determinação do meio de cultura ideal utilizado no cultivo "in vitro", não excluindo os procedimentos usuais como a análise físico-quimica particular a cada meio de cultura.

\section{Agradecimentos}

À FAPEMIG, pela concessão da bolsa de estudos e ao Setor de Cultura de Tecidos da Universidade Federal de Lavras.

\section{Referências}

ALLISON, P.D. Logistic Regression using the Sas System Theory and Application. In: SAS Institute. Books by Users. Washington, 1999.

ANDRADE, L.M.C.O.; PASQUAL, M.; MACIEL, A.L.R.; PEREIRA,A.B.; ALVES, J.M.C. Cultura in vitro de embriões de Coffea arabica influência de NAA e BAP. Ciência e Agrotecnologia, Lavras, v.25, n.5, p.1063-1070, set./out., 2001.

BALAKRISHNAN, N. Handbook of the logistic distribution, Statistics: Textbooks and Monographs, New York: Deker, 1991. v.123.

BUSSAB,W.O.; MORETTIN, P.A. Estatística Básica. São Paulo: Editora Atual, 1987.

COSTA, S.C. Regressão logística aplicada na identificação de fatores de risco para doenças em animais domésticos. 1997. 104p. Dissertação (Mestrado)- ESALQ-USP, Piracicaba.

COSTA, M.P.; PINTO, J.E.B.P.; FRANÇA, S.C.; LAMEIRA, O.A.; CONCEIÇÃO, H.O.; SANTIAGO, E.J.A. Crescimento e teor de emetina em plantas de Ipeca (Cephaelis ipecacuanha A.Richard) obtidas in vitro e submetidas às condições de soluções nutritivas em casa-de-vegetação, Ciência e Agrotecnologia, Lavras, v.24, n.1, p.46-53, jan./mar. 2000.

GONÇALVES, P.S.L. Divergência genética e efeito do nitrogênio total no crescimento in vitro de ipeca (Psychotria ipecacuanha (Brot.) Stokes). 2002. 83p.:I1 Dissertação (Mestrado) - Lavras: UFLA, 2002.

GRATTAPAGLIA, D.; MACHADO, M.A. Micropropagação. In: TORRES, A.C.; CALDAS, L.S.; BUSO, J.A. (Eds.) Cultura de Tecidos $e$

Transformações Genética de Plantas. Brasília: Embrapa-SPI/Embrapa-CNPH, 1998.

MCCULLAGH,P.; NELDER, J.A. Generalized Linear Models, Monographs on Statistics and Applied Probability 37. 2.ed. New York: Chapman \& Hall, 1992.

NANNETTI, D.C. Utilização da cultura de tecidos vegetais na micropropagação e manutenção de Heliconia sp. 1994. 106p. I1 Dissertação (Mestrado) - ESALQ, Lavras.

SOUZA, G.S. Introdução aos Modelos de Regressão Linear e não Linear. Brasília: Embrapa, 1998.

ZIMMERMANN, F.J.P. Introdução aos processos não paramétricos. In: SIMPÓSIO DE ESTATÍSTICA

APLICADA À EXPERIMENTAÇÃO

AGRONÔMICA, 3., REUNIÃO ANUAL DA

REGIÃO BRASILEIRA DA SOCIEDADE

INTERNACIONAL DE BIOMETRIA, 34., 1989,

Lavras. Anais... Lavras: Escola Superior de Agricultura de Lavras, 1989. 
